\title{
Design and Evaluation of an RPL-based Multi-Sink Routing Protocol for Low-Power and Lossy Networks
}

\author{
Kevin Andrea \\ George Mason University \\ 4400 University Drive \\ Fairfax, Virginia 22030 \\ kandrea@masonlive.gmu.edu
}

\author{
Robert Simon \\ George Mason University \\ 4400 University Drive \\ Fairfax, Virginia 22030 \\ simon@gmu.edu
}

\begin{abstract}
Due to the energy and resource constraints of the embedded Wireless Sensor Networking (WSN) devices that form LowPower and Lossy Networks (LLNs), efficient support for mobile data sinks is essential. Further, such support should be fully compatible with the broad class of networking mechanisms now defined for LLN support, such as the routing protocol RPL. This paper describes the Hierarchical network of Observable devices with Itinerant Sinks Transporting data (HOIST) protocol. HOIST is designed to service LLNs using an RPL-friendly three-tier hybrid cluster-tree architecture to support sensor nodes deployed over geographically segregated areas. The mobile aspects of HOIST allow for collection from such remote fields without the need for longdistance wireless transfers, which would result in a higher level of energy drain. HOIST supports both real-time data capture and collected data retrieval. We have implemented and field-tested HOIST in a working physical system, as well as in simulation, with results indicating viability for both sparse and dense deployments of nodes under multiple, geographically constrained, uncontrolled mobility models.
\end{abstract}

\section{Categories and Subject Descriptors}

C.2.1 [Network Architecture and Design]: Wireless Communication

\section{General Terms}

Wireless Sensor Networking

\section{Keywords}

Wireless Sensor Network; LLN

\section{INTRODUCTION}

Wireless Sensor Networks (WSNs) consist of autonomous nodes, which are deployed across potentially large areas according to topological constraints and data collection needs.

Permission to make digital or hard copies of all or part of this work for personal or classroom use is granted without fee provided that copies are not made or distributed for profit or commercial advantage and that copies bear this notice and the full citation on the first page. Copyrights for components of this work owned by others than ACM must be honored. Abstracting with credit is permitted. To copy otherwise, or republish, to post on servers or to redistribute to lists, requires prior specific permission and/or a fee. Request permissions from Permissions@ acm.org.

MSWiM'15, November 2-6, 2015, Cancun, Mexico.

(C) 2015 ACM. ISBN 978-1-4503-3762-5/15/11 ...\$15.00.

DOI: http://dx.doi.org/10.1145/2811587.2811614.
These nodes collect information about their environment from attached sensors. As WSN nodes are typically powered either by batteries or through energy-harvesting, these devices are power-limited, necessitating the use of resourceconstrained, embedded systems. These devices operate over a type of network called Low-Power and Lossy Networks (LLNs), which feature high loss rates, instability, and low data rate transfers[1]. Such WSNs are presently used in precision agriculture[2], disaster management[3], and industrial monitoring[4], where they are expected to operate for extended periods of time without the ability of network operators to perform routine maintenance.

This paper describes Hierarchical network of Observable devices with Itinerant Sinks Transporting data (HOIST) protocol and architecture. The underlying design philosophy of HOIST it to be fully compatible and inter-operable with existing LLN networking standards. It is widely recognized that resource constrained LLNs requiring convergecast routing (a characteristic of our targeted application class) gradient routing techniques are particularly effective [5]. In recognition of this fact, the IETF has standardized the Routing Protocol for Low Power and Lossy Networks (RPL) protocol [6]. HOIST is designed as an application layer control plane semi-reliable protocol to be used with off-the-shelf RPL implementations.

HOIST provides a means for data collection from WSNs in a manner that is able to handle such high loss rates, network instability, and low data rate transfers as is expected with LLNs. Building upon basic research in mobile sink support via hierarchical topological methods [7, 8], HOIST is a hybrid tree-cluster, three-tier hierarchical architecture that coordinates both data relay and data sink collections from multiple, geographically segregated WSN deployments. We implemented HOIST with both physical devices and in simulation, and we have analyzed control message timings for enhancing initial deployments. One of the most difficult challenges in supporting mobile sinks is to reduce the amount of time for the sink to establish stable multi-hop routing paths in the networks being visited. As will be seen, a primary focus of our work is to precisely quantify, in our experimental prototype, the time required to establish such routes.

HOIST utilizes uncontrolled mobility to support motivating applications such as precision agriculture[2]. In this application, there can be multiple, geographically segregated fields that are covered with WSN devices. Using mobility, HOIST enables mobile data relays to move between fields to collect stored sensor data. When this data relay arrives 
at each field, it can follow geographically constrained pathways as dictated by the terrain and WSN field applications themselves. This data relay may also be deployed on aerial vehicles that are able to enter and hold an orbit around each field for the duration of the data collection.

At network startup, the lower-tier of HOIST sensing devices begin their collection from the environment. These nodes will locally store their data in non-volatile memory, without any data transmissions during this normal state of operations. The middle-tier devices are local sinks that are manually placed in a linear topological formation, throughout the center of the node deployment area, and act as cluster-heads for the lower-tier nodes. These middle-tier sinks provide routine node control and network maintenance functionality while awaiting the arrival of one, or both, of the mobile sinks to the area.

The upper-tier devices consist of mobile sinks in either a data relay or a data sink role. This allows both retrieval of collected data from each field, as well as an immediate assessment of the field by performing a spot-check, without interrupting either current or future retrieval operations. When a mobile sink enters range of any middle-tier sink, it will initiate the creation of a tree-like backbone connecting each of the local sinks. Once this is formed, the local sinks coordinate their subordinate nodes to transmit their data. For the mobile data relay, nodes send a number of messages based on network conditions before seeking verification of data delivery. Once this verification is complete, the nodes will continue their broadcast until data transmission is complete. Each of these transmission processes is such that each sink is only coordinating a single sensor device transmission at a time. This reduces network flooding, allowing for sensor device scaling with a logarithmic order of growth in message traffic, as concurrent data transmissions scale linearly with the middle-tier sinks, not lower-tier collectors.

\section{RELATED WORK}

The aim of HOIST is to provide a means of collecting data from geographically segregated fields of sensing nodes. Topological approaches for mobile sink support have been very heavily studied in wireless networking literature $[7,8]$. For instance, there has been much work showing the benefits of both cluster and hierarchical based architectures. Clusterbased architectures $[9,10]$ divide nodes into virtual groups that limit hop-count for data transmissions and either reduce the overhead of node control messages or the overhead of data forwarding. These approaches designate certain devices as cluster heads, whose responsibility includes the coordination of data transfers and overall management of subordinate devices. Hierarchical-based architectures[11, 12] provide scalable data dissemination through multiple tiers of routers, often using a backbone line, to reduce the number of query and data transmissions between a node and the data sink.

We now describe in detail several relevant architectural approaches. Hierarchical Cluster-based Data Dissemination (HCDD) [12] is an architecture that leverages the benefits of clustering with a hierarchical model by designating certain nodes as routing agents. Sensor nodes first send their data to the local routing agent, which then forwards the data through the interconnection of routing agents towards the sink. HOIST makes use of a similar model by using a middletier of local sinks to act as routing agents. Using RPL as our routing interface, each sink is the root of its own DODAG; by placing these sinks in a linear topological formation across the center of the deployment area, we enjoy the benefit of clustering through lower hop count DAGs.

The Hierarchical Role-based Data Dissemination (HRDD) [11] architecture alleviates energy drain problems through mobility, while likewise using clustering and a data dissemination backbone to send data to the sink. The primary achievement of HRDD was alleviating energy-usage problems, reducing the quantity of data and query transmissions, and accelerating the data delivery. One key feature with HRDD is that nodes only forward metadata about events of interest. If the sink desires the full report, it will send a query down for the full data. In HOIST, middle-layer sinks provide all of the route maintenance and coordination functions, similar to the cluster heads. When the mobile data relay arrives, it sends an announcement of arrival to the middle-tier sinks, which form a backbone and begin coordinating with each of their subordinate sensor nodes to send their full data. Each middle-tier sink coordinates the transfer of each node, one at a time, to prevent undue flooding of the network.

A multiple-tiered model with ferrying sinks has been implemented by [13]. Their Mobile Ubiquitous LAN Extension (MULE) approach is designed for sparse sensor networks that enable data to be locally collected and stored before being transmitted to its middle-tier wandering sink devices. HOIST differs in that our sensor nodes form a static network within the middle-tier, maintaining a routing network before the mobile sink arrives. Once any of the mobile sinks arrive, nodes send data through pre-existing routes, removing the need for dynamic routing on the lower-tier in response to any mobility.

[14] describes a three-layer system in an approach called Load Balanced Clustering and Dual Data Uploading (LBCDDU). In this approach, a clustering algorithm elects cluster heads from amongst the collectors, such that the collectors are all one-hop from a cluster head. The cluster heads coordinate data with the top layer, which is a mobile sink called a SenCar that plans a route to directly collect data from each cluster head. Mobile sinks in HOIST only have to be within range of any of the middle-tier sink devices to communicate with the entire network. In many cases, such as in an agricultural deployment, terrain may prevent direct connection with each cluster head, so HOIST allows mobile devices to skirt along the region and manage communications from the perimeter.

Data sink primary networks for spot-checking conditions without interrupting existing applications have also been described in use. [15] describes how WSNs may be used in urban environments with the ability for emergency responders to connect and view current building sensor information. As each firefighter arrives on scene, their local devices connect with the building and begin receiving sensor information. HOIST employs a mobile data sink for a similar purpose, enabling any end-user to view current conditions in a deployment area.

[3] uses a large WSN deployment to provide an alert service to first responders for fire outbreaks in cultural heritage areas. Their technique focuses on redundancy by sending data to multiple sinks. In HOIST, multiple sinks are used in a similarly hierarchical manner to increase the scale of the deployment while still retaining the ability of the mobile sink 
to reply directly to any node in the network. This ability to reply enables data assurance, allowing nodes to retransmit data until delivery is acknowledged, mitigating the need for such redundancy.

Further, mobility models for mobile data collection has also received much interest [7]. Mobility can either be controlled, in which the network moves sinks predictably according to a known process, or uncontrolled[16], with processes beyond the control of the network driving the movement of the sinks. Mobility is also used to reduce the energy cost of networks by either moving directly adjacent to cluster heads [13], or by more evenly distributing the transmission load on a convergecast routed network. In convergecast, devices closest to the sink have to retransmit a disproportionately large number of messages, expending more energy in a phenomena known as the hot spot problem[17]. Mobility addresses this problem by allowing sinks to move about the network, more evenly distributing the power expenditures. As mentioned above, HOIST assumes the most general purpose uncontrolled mobility model.

Finally, due to its importance in practical network settings, RPL support for mobile applications has recently received much attention $[18,19,20]$. However, to the best of our knowledge, HOIST represents the first evaluation of an implemented hierarchical approach for mobile sink support that is fully compatible with standard RPL.

\section{ARCHITECTURAL DESIGN}

In HOIST, we designed a three-tier architecture to implement a hybrid tree-cluster design, using uncontrolled mobility and the common RPL protocol to provide routing support. HOIST was designed to be used in either sparse or dense networked environments, without any direct lines of communication to a fixed collection center. We utilize specially designated mobile sink devices in the two primary roles for remote data collection: data sink and data relay. The data sink fills the traditional model wherein sensor devices send their information directly to a sink, as the data is collected. A data relay, however, serves the role of moving between the WSN fields to collect data. Not only does this mitigate problems pertaining to energy drain, which would result from trying to relay data from distant fields, but it also enables us to have multiple, independent and geographically segregated fields of deployments in simultaneous operation.

HOIST is an application-layer protocol that governs the coordination and data transfers between the devices at each tier. For the data forwarding between each of the devices, we rely on the RPL protocol. RPL creates and manages routing for the network by using its own ICMPv6 control messages. Messages in HOIST are transmitted as data under the UDP transport protocol; however, these HOIST control and data forwarding messages also use RPL options at the IP layer, which allows RPL to govern the forwarding. RPL specifies this forwarding through the use of the RPL Instance identifier, which itself addresses the root of a collection of DODAGs. HOIST uses three such RPL Instance identifiers for routing; as these identifiers are single-byte fields, we have elected to assign them mnemonically using single ASCIIencoded characters. These identifiers are introduced along with the HOIST sinks below.

The fundamental feature of HOIST is this hierarchical design, as shown in Figure 1, using three tiers of nodes. At the lower-tier, sensor devices known as Collectors sense fea- tures of their environment and store such data locally for later retrieval. The middle-tier nodes, known as Bridges, are the local sink devices that perform the role of cluster-heads. These Bridges are each sinks within RPL Instance identifier 'B', opening themselves up for Collectors to join them. Collectors are able to switch between Bridges as network conditions suggest. The Collectors are completely insulated from any network changes made by any higher-tier mobile sinks, reducing the time necessary to update the network topology following mobility to the Bridge sinks only.

The final, upper-tier of HOIST consists of two discrete Instances. Instance ' $\mathrm{M}$ ' is created by a mobile data relay sink called the Messenger, whose objective is to travel to each of the remote WSN deployment fields, retrieve the collected data, and return such data for processing. When the Messenger arrives at a deployment site, RPL will initiate its own route creation by sending a DODAG Information Object (DIO) message, which HOIST uses to indicate mobile sink arrival. When this DIO is received by a Bridge, it is rebroadcast to cause all of the Bridges to create a multi-hop DODAG. Once connected, the Bridges coordinate with each of their descendants, one at a time, and act as the relay to send their data to the Messenger. The total number of Collectors sending data is limited to the number of Bridges, reducing the network load. As a Collector finishes its data transmission, it immediately resumes collecting data.

The second Instance supported by this upper-tier is Instance 'O', which is created by a mobile data sink called the Observer. Like the Messenger, this sink can enter the deployment area at any time, causing all of the Bridge devices to join to that Instance. Once connected, however, the Bridges signal each of their Collectors to send the Observer a copy of their latest data. The Collectors will then send a copy of each new piece of data, as it is collected. In this manner, the Observer is able to perform a spot-check on the network.

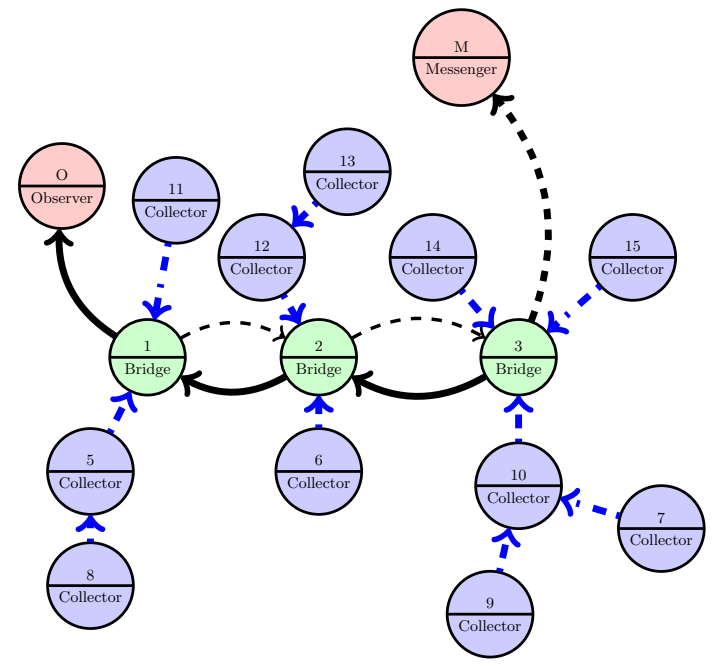

Figure 1: HOIST Overview

In HOIST, Collectors are deployed statically as needed to monitor the local area; they are not affected by any network topological changes stemming from mobility. Once the Collector deployment is completed, Bridge devices are introduced to act as coordinating cluster-heads for the Collectors. 
This deployment is done manually by placing them in a linear topological formation such that the Collectors each have a valid multi-hop route to least one Bridge, and that the Bridges are able to evenly distribute the load of Collectors.

The Bridges are all sinks for RPL Instance 'B', which is the collection wrapper for all Bridge DODAGs that RPL uses for its routing. Aside from this function, the Bridges do not collect any data themselves. HOIST uses a linkquality aware Objective Function (OF) with hysteresis for these RPL DODAGs to promote stable, efficient routing. This does not need to be the OF used for either of the mobile sinks; they may use their own custom objective functions as best fits their mobility models.

\subsection{HOIST Protocol}

HOIST introduces several message types to facilitate coordination and data transmission between the myriad tiers. These messages are sent as application data in UDP packets, routed using IPv6 and RPL. These are separate from the ICMPv6 network control messages used by RPL. The primary messages HOIST introduces are described below.

SYN Sent by Bridges to determine when bi-directional routing is established with the Messenger, which sends a SYN in reply to the originating Bridge.

START Sent by Bridges to coordinate Collectors to begin data transmissions.

DATA Sent by Collectors and relayed by Bridges to send data to the mobile sinks.

CHECK Sent by Collectors to query the Messenger for the last expected message.

ACK Sent by the Messengers in reply to CHECK.

FIN Sent by Collectors to inform their Bridge their data transmission is complete.

TERM Sent by Bridges to inform their Collectors a mobile sink has left.

These messages are sent as application data, carried by UDP, using RPL for its routing. The HOIST data frame is depicted below. Type refers to the HOIST message types above. Bridge ID is used by the Bridge to record the last octet of its IPv6 address. Collector ID is the originating Collector's last octet of its IPv6 address, which is used by the Messenger for data storage purposes. $S R$ is the send rate of the Collector and $D C$ is the number of data fields in this message. Starting Index provides the starting index of the data from the Collector, which is used by the Messenger for data assurance. Data $[x]$ refers to the 2-byte data fields that follow.

\begin{tabular}{|c|c|c|c|c|}
\hline HOIST Type & Bridge ID & Collector ID & SR & DC \\
\hline \multicolumn{2}{|c|}{ Data $[0] \ldots$} \\
\hline
\end{tabular}

One of the primary factors of HOIST is data assurance for the Messenger device. We have elected to implement a hierarchical network capable of enabling the Collectors to request the next data message expected by the Messenger. This enables the Collector to confirm delivery receipt and schedule any repeated messages as needed to assure complete data reception by the Messenger. HOIST presently performs verification for contiguously received data, which means a Collector will need to resend data beginning with the first message that was not received by the Messenger. The number of messages a Collector may send between these verifications is autonomically adjustable through HOIST.

Communication begins once the initial RPL configuration message (DIO) for Instance 'M', formed by the mobile collection sink, is received by a Bridge as shown in Figure 2. Once this occurs, the receiving Bridge will generate a SYN message for the Messenger. The naive problem with immediately coordinating data transfers is that, for more distant Bridges, the RPL routing table construction messages will not have had ample opportunity to propagate back to the Messenger, leaving the Bridge unroutable. To prevent this cold-start routing miss, a simple SYN handshake takes place. Once the Bridge receives a reply, it begins data coordination. It is normal for this SYN message to timeout on several attempts prior to the first successful reply.

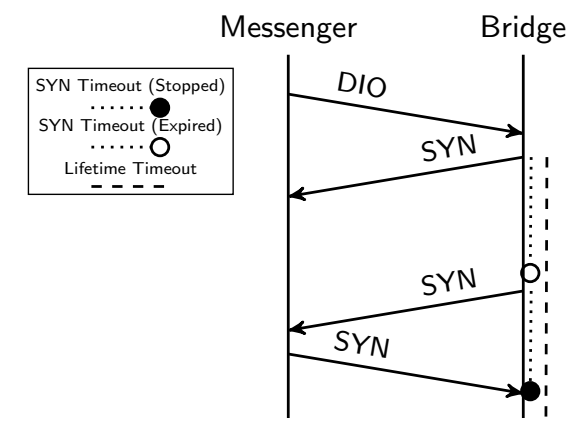

\section{Figure 2: Arrival Handshake - With Timeout}

As shown in Figure 2, there are two timeout mechanisms governing this process. The first enables the Bridge to send another SYN packet to the Messenger. The second is a lifetime timeout. This concept of lifetime, described in section 3.2 below, is an enhancement we made to our RPL implementation to signal HOIST that a mobile sink is gone. Should a mobile sink leave (or cease functioning) during the initial SYN process, the Bridge will cease attempts and resume normal operations.

Once the reply SYN message is received, a START timeout period is started and the Bridge begins by sending a message to the first Collector in its downward routing table. That Collector replies with the same message in acknowledgment, after which the Bridge begins waiting for data traffic to relay. If a Collector is unreachable, the timeout will occur, supporting up to $\mathrm{N}$ attempts at communications before moving to the next device in the list. Once a Collector receives the START message, it will begin sending its data in packets, maximizing payload data without fragmentation, as shown in Figure 3.

Once a Collector has received this message, it sends blocks of messages to the Bridge using RPL Instance 'B' for addressing, starting a timeout on the Collector. When a Bridge receives a data packet, it sends it using RPL Instance 'M' for addressing. Upon receipt by the Messenger, the data is examined to determine if it is the expected next block. If so, it adds the data to persistent flash memory for later retrieval and updates its next expected block information.

A Collector will always send at least one block of data towards the mobile sink during its transmission operation. 


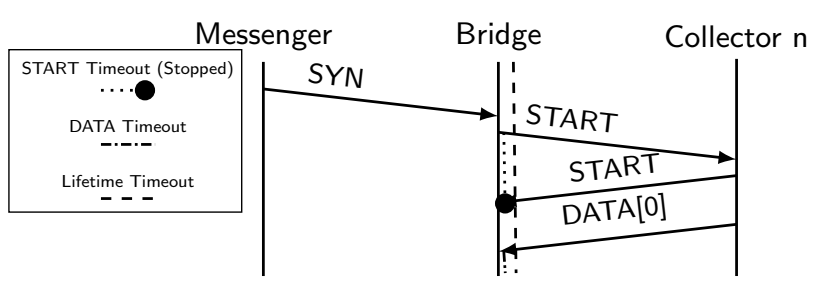

Figure 3: Data Initiation Handshake

HOIST enables Collectors to adjust the number of data blocks they are able to send in response to link quality information, by timeouts encountered, or through hardcoding, as desired. When a Collector has either sent all of its data, or has sent the maximum number of unconfirmed blocks, it will send a CHECK message to the Bridge. The Bridge forwards this to the Messenger, which will immediately reply back with an ACK message containing the next expected message. If the next expected message matches the next message to send, then the Collector will proceed with either finishing the connection, or it will begin sending the next set. This process is depicted in Figure 4.

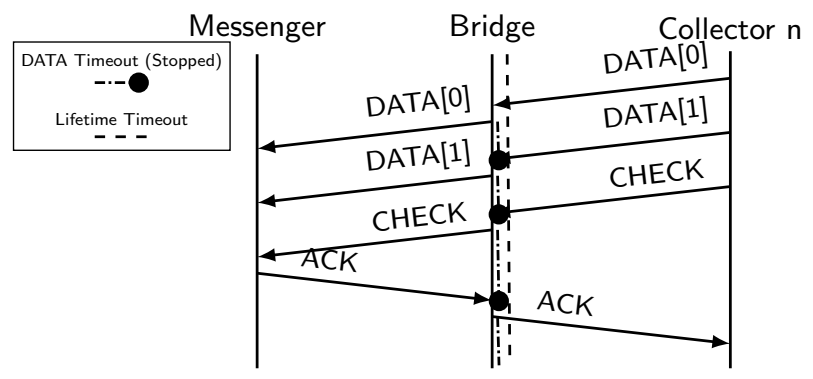

Figure 4: Data Transfer

If the Collector receives no response from the CHECK message, it will continue to resend CHECK packets. HOIST uses two different timing values for this CHECK message retransmission. For the first set of failures, HOIST uses a short duration timer for resending these messages. This is done to account for dropped packets that occur with any LLN communications. After the first set of failures, the Collector operates on the assumption that the Messenger is out of range and routing needs to be rebuilt. At this point, the Collector uses a longer timer, which is designed to give RPL the time to rebuild routing to the Messengers new location. Once an ACK is received, the Collector resumes data transmission operations. Should the Messenger leave communications range long enough that lifetime expires, then the Bridge sends TERM to the current Collector, as shown in Figure 5. When received, the Collector resumes collection operations, marking the last successfully transmitted data segment so it may later resume where it left off.

When the Collector has sent all of its data and has received the ACK message with the proper confirmation, then it will issue a FIN message the the Bridge. Upon receipt, the Bridge will reply with a FIN message back to the Collector and then send a START message to the next Collector in its downward routing table. This process will continue until either all Collectors have finished, or until the Messenger leaves the area and its lifetime expires, at which point the ac-

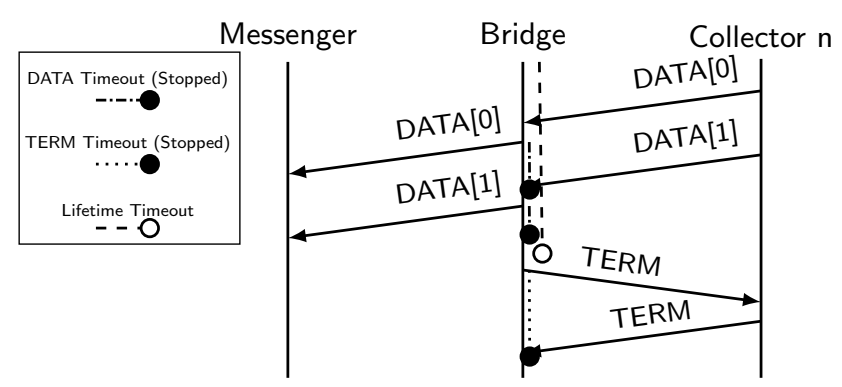

Figure 5: Data Transfer - Lifetime Timeout

tive Collector will be terminated and the Bridge will resume normal operations. All Collectors not actively in sending data to the Messenger will be in normal collection operations and will send their data to the Observer, if present.

The Observer is implemented similarly to the Messenger, albeit without any message delivery assurance. HOIST makes this distinction for the primary reasons that the $\mathrm{Ob}-$ server is a traditional data sink that is interested in the current state of the devices in the local area. Any lost messages will be replaced when a Collector sends following its next collection event. The secondary reason for the lack of delivery assurance is to reduce the need for additional HOIST control message overhead. As the Observer may be present along with the Messenger, adding CHECK and ACK messages would contribute to the flooding on the order of the number of Collectors. As the network operates on the order of the number of Bridges for Messenger operations, this would tremendously increase the flooding of the network; as such, the Observer operates without data assurance.

When the first DIO for Instance 'O' is received by a Bridge, it forms a multi-hop DODAG with the other Bridges, with the Observer as root. Each Bridge then sends START to each Collector. The first messages to the Observer will occur directly after receiving START, consisting of the most recent data collected by the Collector. After this, when a Collector normally collects data on the environment, in addition to storing the data in its own internal array, it will also send a copy through the Bridge to the Observer. This process will continue until the Observer leaves the network, at which point the Bridge will send TERM to each Collector. The Collectors then will continue normal operations.

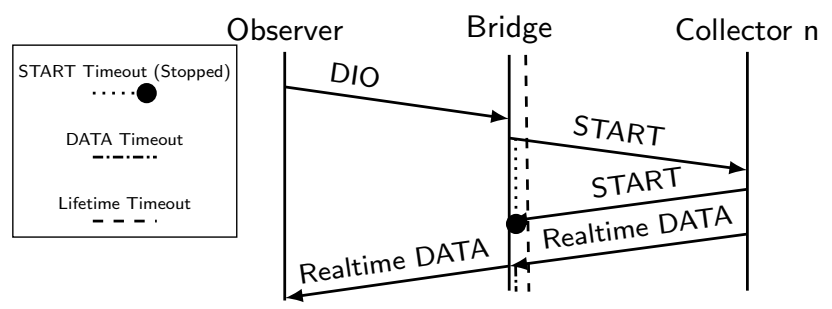

Figure 6: Data Initiation Handshake (Observer)

In the case that both the Observer and Messenger are in range simultaneously, the network will operate as it would for either independent event, with one exception. While a Collector is sending its archived data to the Messenger, it will not be collecting any new data; this means that no messages will be sent to the Observer from that Collector. 
Once the data send terminates and the next Collector in the same DODAG begins sending to the Messenger, then the previous Collector will resume collection operations and will send to the Observer.

\subsection{RPL Protocol Enhancements}

HOIST specifies the mobility, topological, and messaging designs, however, it interfaces with the Routing Protocol for Low-Power and Lossy Networks (RPL)[?], the IETF standard for LLN routing, to handle lower-level message forwarding between the various devices in the network. HOIST uses RPL to create, manage, and provide inter-device routing for HOIST messages. RPL manages routing through a tree-like Destination-Oriented Directed Acyclic Graph (DODAG) structure, which HOIST uses for all of its message forwarding.

As RPL is an IP layer extension, its primary role is to provide routing support for IPv6 message delivery. The primary concern with respect to HOIST is that RPL does not have any inherent support for mobility, leading to network partitioning when the mobile sinks move, while RPL works to identify and correct its routing. While RPL has no control message parameters to notify arrival or departure of a device to a network, it does send the DIO control message from the mobile sinks to initiate DODAG construction. HOIST uses the arrival of a new DIO message as its indicator that a mobile sink has arrived.

While HOIST is able to extract arrival information, RPL has no support for determining departure of a sink from the area. RPL does have a lifetime field governing its instances, however, the lifetime on an Instance only affects routing to that Instance. Once this mechanism is exhausted, the Instance remains active and DIO messages continue to be generated concerning this Instance, which continue to propagate out-of-date information. With no means of knowing if this Instance is alive or not, the Bridges are unable to inform the Collectors of departure and therefore no means for them to resume normal collections.

We enhanced the lifetime mechanism of RPL to extend it for Instance lifetime governance. DIO configuration messages in RPL contain specifications for the routing lifetime of the instance. We elected to use this lifetime information from the mobile sinks as an Instance lifetime counter, which is locally stored on the Instance structure. When a DIO is received, the lifetime is read from the configuration option and a timer is started. When that counter reaches zero, the Instance is purged from the device, which HOIST uses as the indicator that the mobile sink has left the area. When a Bridge propagates a DIO message from a mobile sink to other Bridges - a continual routing maintenance function of RPL - it will send its own current lifetime for the Instance, not the default initial lifetime. This has the advantage of equalizing the lifetimes of the network of Bridges amongst each other. Once a mobile sink either leaves range, or dies, the Bridges will continually decrement their lifetime values for that sink's Instance until it reaches zero, at which point every Bridge simultaneously removes the Instance. This prevents out-of-date information from propagating across the network and makes the Bridge ready for the next mobile sink to arrive.

The second enhancement we made was necessary to support the hierarchical nature of HOIST. In RPL, any DIO received by a node will be accepted as an Instance and joined. This would pose severe problems in situations where a node might attempt to join the 'M' or 'O' Instances directly, should these devices be active in range of a Collector when it comes online. To prevent this, we implemented both a Whitelist and a Blacklist system in RPL, which are configurable in the code for each of these device types. In HOIST, Collectors have only one authorized Instance in their Whitelist: Instance 'B'. Bridges, on the other hand have Instances ' $O$ ' and ' $M$ ' in their Whitelist. We implemented this system in the algorithm processing DIO arrival messages. Upon arrival, if either Whitelist or Blacklist constraints are defined, the Instance ID is compared and the DIO is either rejected or accepted as specified.

\section{HOIST IMPLEMENTATION}

We have implemented HOIST under the Contiki 2.7 Operating System, modified to support multiple RPL Instance routing, Instance whitelisting and blacklisting, and communally balanced RPL Instance lifetimes. While HOIST is easily implementable at the application level, our modifications to RPL needed to be modified within the operating system networking core. As the Contiki operating system provides a base implementation of RPL, we also needed to make some minor changes to complete their implementation. RPL, as implemented by the Contiki operating system, only supports single-Instance RPL networks. We provided significant modifications to the core networking code to enable multi-Instance networks to form. The first modification was to extend the rpl_dag table to support storing DODAGcentric metric information for parent selection. Previously, this was a global table that stored one rank for each neighbor, regardless of Instances; this prevented all possibilities of operating in a multi-Instance environment. Compounding this, several functions, which were written with the innate assumption that only one Instance or DODAG would ever be in existence at a time, had to be modified.

The core routing decision code also needed to be modified. In Contiki, a four step decision process is executed to determine the proper next hop for the message. For upward routing towards the root, this meant that, regardless of the message's stated Instance ID, all messages destined for non-neighbor sinks would be routed to the default Instance's preferred parent only. This default Instance, however, is set by incoming DIOs, meaning this was like sending a message on a raft down a river that changes directions arbitrarily. While this technique is sufficient for single-Instance routing, any attempt at routing within a multiple-Instance environment will result in failure.

We modified this by adding a step immediately after checking the downward routing table, which is shown as the italicized step 4 in the following list. This additional step checks the RPL Instance ID on the message received and then uses that Instance's preferred parent for routing instead. This modification additionally involved writing many functions to perform such checks. This is a new type of forwarding to facilitate proper routing in a multiple RPL Instance environment.

1. Determine if the destination address is multicast. If the address is a multicast address, send the message.

2. Otherwise, check if the destination address is in the neighbors table. If so, send the message as addressed.

3. Otherwise, check if the destination address is in the 
downward routing table. If so, send the message to the next-hop address as listed in the table.

4. Otherwise, check the destination RPL Instance ID on the message. Send the message to the preferred-parent of the Instance specified on the message.

5. If there is no RPL header, or if the Instance is unknown, send the message to the preferred-parent of the default Instance.

In this extension to the RPL implementation, the message will be routed as addressed in the event that it is not an adjacent destination. If the Instance is not known, then the message will still be sent along the local device's default Instance to continue to make progress in the understanding that the next device may have a viable route. Messages with no RPL headers are also assessed in this stage to see if their destination address matches a known RPL Instance sink. If so, an RPL header is added to more rapidly facilitate proper routing.

One of our choices in HOIST utilizes the ability to select a custom objective function for each RPL Instance. The current implementation environment on Contiki required a minor modification to enable each device to recognize the full gamut of possible objective functions. We are presently assessing different objective functions at each level for various platforms of mobility. We have tested HOIST using an Unmanned Aerial System (UAS) as the platform for our mobile sinks and discovered that using a hop count based objective function for the mobile sinks was effective when the UAV orbited the entire field, frequently entering and leaving the radio range of each Bridge.

\section{HOIST TESTING}

We have designed these tests to validate HOIST through the analysis of several factors, and their responses. The primary factors of interest in developing baseline settings for HOIST are the DIO minimum send rate, the maximum DIO send rate doublings, the data transmission send rates, and the number of Collectors in the network. We are interested in these values particularly as they govern the timing for sending RPL's DODAG formation and maintenance control messages. These messages also affect the responsiveness of RPL to mobility. If too few messages are sent, then hysteresis on the Bridges will prevent them from adopting the new routes of a sink after it has moved; however, if they are too frequent, then the network may be inundated with messages that do not offer any new information. As mobility is present in this network, we have designed the tests to ensure these DIO related factors are examined in situations that include network partitioning and reformation. Only by ascertaining a baseline for RPL timings relating to message routing, can we better assess efficacy of HOIST under multiple scenarios.

\subsection{Physical Validation}

We conducted the first physical validation on nine Zolertia Z1 motes using one Messenger, three Bridges, and five Collectors. A close-up showing a representative set of these devices is shown in Figure 7.

For this implementation in Figure 7, we initialized each Collector with 30 data samples, necessitating each to transfer two data packets to the Messenger data relay, by way of the Bridges. The network formation is visible through LED

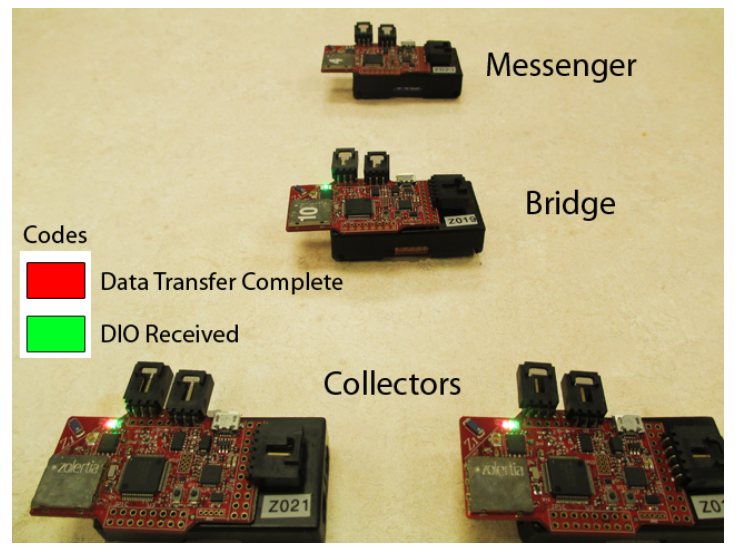

Figure 7: Subset of Physical Implementation

codes on the devices. As each node receives its initial DIO, the green LED illuminates. As each Collector finishes its data transmission following the final successful ACK reply, they illuminate their red LED. This trivial demonstration shows HOIST operational on physical Zolertia Z1 devices.

\subsection{Simulation Testing}

We have used the Cooja simulator to perform baseline analysis on HOIST, with the aim of ascertaining initial settings and suitability for future collection operations. The first round of testing uses 8 Collector and 4 Bridge devices to determine ideal settings for the DIO transmission rates to optimize building and maintaining the HOIST network. For this testing, the Bridges are deployed linearly, with each placed at a distance of $70 \%$ of the maximum transmission range, with an offset equal to a random value, sampled from the Gaussian distribution (generated using the Box-Muller method[21]) with a variance of $5 \%$ of the maximum transmission range to ensure only its adjacent neighbors are in range. The Collectors are generated after the Bridges and are placed at a distance equal to $70 \%$ of the maximum transmission range of an existing device, with an offset equal to a Gaussian random value with a variance of $5 \%$ of the maximum transmission range. The angle of placement from the center of the existing device is sampled uniformly.

\subsubsection{Simulation Mobility Model}

For the first mobility model, we used a geographically constrained path to a point adjacent to the farthest Bridge in the linear formation. The Messenger sink (M) begins at a distance of $150 \%$ maximum transmission range away from the first Bridge (B2). Following 30 seconds, the Messenger begins moving at $1.3 \mathrm{~m} / \mathrm{s}$ and progresses until it is directly adjacent to the last Bridge (B5), where it halts and remains until the end of the simulation. This test is designed to ensure the Bridges form an initial network through B2 and begin data transmissions, that the radio connection between $\mathrm{M}$ and B2 breaks due to exceeding the maximum transmission range, and the network has to ultimately reform once $\mathrm{M}$ stops. These initial positions and this movement are depicted in Figure 8.

The second mobility model is used for the orbit tests to emulate an Unmanned Aerial System (UAS) in flight, orbiting around a field of deployed devices. In this model, the Messenger sink begins in the same manner, however, 
once it has entered the field, it moves at a pace of $5 \mathrm{~m} / \mathrm{s}$ along a geographically constrained path between the first and last Bridges, simulating an orbiting vehicle. This test is designed to force rapid routing changes to continue data transmissions.

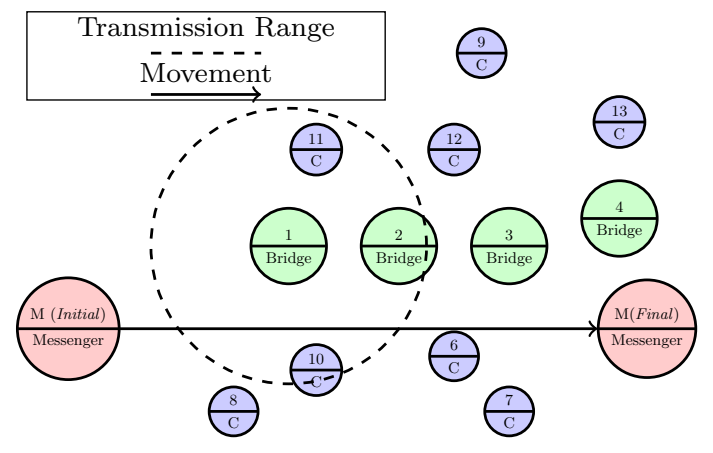

Figure 8: Mobility for Validation

\subsubsection{Analysis of DIO Settings}

For the first tests of HOIST, we seeded each of the 8 Collectors with 30 data points and set their node collection rate to 120 seconds. The Messenger begins moving at 30 seconds and ceases its movement when it is adjacent to the last Bridge. We ran 30 simulations for each combination of settings for the DIO transmission rates. We selected minimum times for DIO transmission to $2^{11} \mathrm{~ms}, 2^{12} \mathrm{~ms}$, and $2^{13}$ ms, and tested each with 4,5 , and 6 doublings of the Trickle algorithm[22].

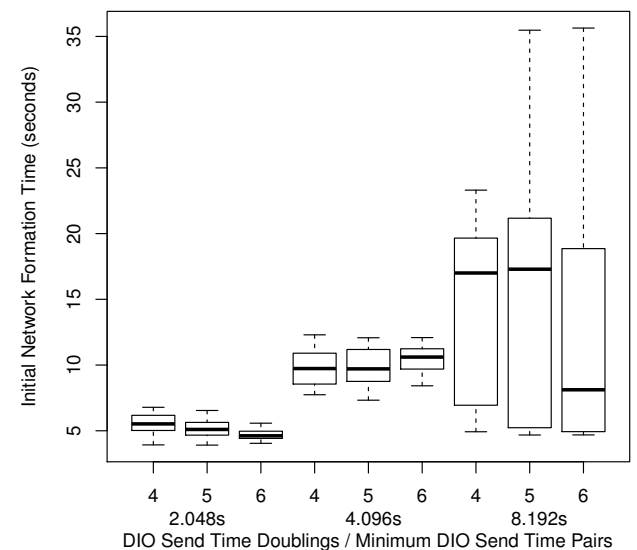

Figure 9: Initial Formation Time Analysis

The collected data for the initial formation time of the 'M' Instance is shown in Figure 9. Using two-factor ANOVA analysis with replication, we calculated that, with a $95 \%$ confidence level, using a minimum DIO send time of 2.048 seconds was statistically better at reducing initial formation time, whereas the number of doublings in send rate, through the Trickle algorithm, had negligible effects. There were no statistical interactions between the two factors.

With respect to the duration of the data transfer, we performed two-factor ANOVA analysis with replication, shown in table 2. Looking at the data transfer duration, we did not observe any statistical difference in data transfer times

\begin{tabular}{c|c|c|c}
\hline Source & F-Statistic & F-Critical & P-Value \\
\hline DIO Minimum & 10.34589 & 3.89546 & 0.001547 \\
DIO Doublings & 1.6970 & 3.0479 & 0.1863 \\
Interaction & 1.7137 & 3.0479 & 0.1832
\end{tabular}

Table 1: Two-Factor ANOVA on Formation Time

due to the minimum DIO send time, however, the number of doublings was significant at a $95 \%$ confidence level. This is as expected insofar as these simulations partition completely all routes to the Messenger early in the transfer of data. Reducing the maximum number of doublings causes the Messenger to send more DIO messages, which help to break hysteresis and enable Bridges to directly connect and restore routing.

\begin{tabular}{c|c|c|c}
\hline Source & F-Statistic & F-Critical & P-Value \\
\hline DIO Minimum & 0.00098 & 3.8955 & 0.9751 \\
DIO Doublings & 3.2270 & 3.0479 & 0.0421 \\
Interaction & 9.1561 & 3.0479 & 0.00017
\end{tabular}

Table 2: Two-Factor ANOVA on Transfer Duration

\begin{tabular}{c|c|c|c}
\hline Source & F-Statistic & F-Critical & P-Value \\
\hline DIO Minimum & 2.3747 & 3.0304 & 0.0951 \\
DIO Doublings & 3.8977 & 3.0304 & 0.0215 \\
Interaction & 1.5993 & 2.4062 & 0.1749
\end{tabular}

\section{Table 3: Two-Factor ANOVA on Route Reforming}

Examining the data collected, we found a great variance on the data transfer times, which appear to have more to do with the placement of the devices than the settings used. We further saw the time it takes to reform the network after initial partitioning was quite similar in that, under RPL, the number of runs where the routing failed to fully reform as of the 600 s end of the simulation varied greatly.

For this analysis, as seen in Table 3, we see that while the results vary greatly, the minimum DIO send time did have a statistically significant impact; however, both $2.048 \mathrm{~s}$ and $8.192 \mathrm{~s}$, for these scenarios, yielded similar results with respect to both reform duration and in the number of reform completions. The final assessment performed was on the number of repeated packets necessary to ensure full delivery. Combining the data on the myriad assessments, we have opted to select a minimum DIO send time of $2.048 \mathrm{~s}$ and 6 doublings as the baseline for our data collection in the presence of a mobile sink.

\subsubsection{Analysis of Send Rates}

We next worked to establish a baseline for selecting the send rate of the Collectors. This rate governs only the data transmissions, however, this factor is important to properly tune to ensure prompt, but reliable message delivery in increasing network densities.

For these tests, we used the same configuration for the Bridges, however, the Collectors will now vary in size between 8 and 12 devices to increase load on each Bridge. To focus only on the contribution from the Collectors to this analysis, we now stop Messenger mobility when it is adjacent to the first Bridge to prevent network partitioning. For these runs, each combination was repeated 30 times, using 
a DIO minimum send time of $2.048 \mathrm{~s}$ and a maximum of 6 doublings.

The first test of significance is to explore the relationship between the send rate and the number of Collectors in the network. We performed two-factor ANOVA analysis results for every combination of 8,10 , and 12 Collectors under 2, 3 , and 4 second send rates. With respect to the number of Collectors, we found there was a statistical significance, at a $95 \%$ confidence level, with a p-value of 0.0813 , that the chosen send rate did affect the data transmission times. This warrants further testing under larger networks of devices; however, it shows that even in the presence of increasing local density, a smaller send rate still provided benefits overall.

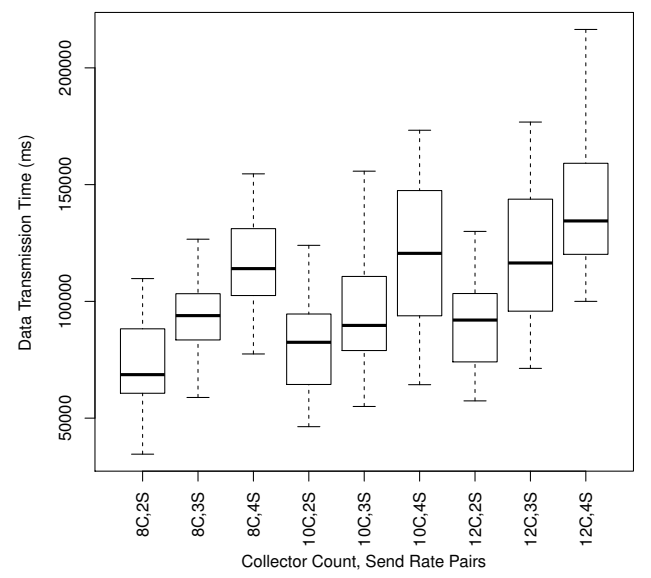

Figure 10: Send Rate Analysis

\subsubsection{Orbit Mobility Analysis}

Our final validation testing was conducted using the Messenger sink aboard an Unmanned Aerial System (UAS), flying in an orbit around a HOIST deployment field. We conducted an initial test using Zolertia Z1 sensor devices aboard an Aurora Flight Sciences Skate UAS[23]. Our integration with the Skate UAS is shown in the cut-away of the payload bay in Figure 11. Our initial testing was conducted with the top two tiers of HOIST only, to establish multi-hop connectivity with RPL using the MRHOF objective function.

We performed initial validation using a simple program that broadcast UDP packets and recorded signal strength received. We programmed the Skate to fly in a $15 \mathrm{~m}$ orbit at $30 \mathrm{~m}, 23 \mathrm{~m}$, and $18 \mathrm{~m}$ altitude above the ground. The wind for this flight was $4 \mathrm{kts}$ with gusts to $10 \mathrm{kts}$, which caused the aircraft to fly in a highly irregular orbit. Despite this, we received approximately $80 \%$ of the messages sent from the UAS at the lowest altitude, which we used to fly all subsequent tests.

Our main validation flight tested multi-hop routing between the upper two tiers of HOIST, with three bridges statically deployed on the ground and the Messenger carried aboard the UAS. Our deployment of three Bridges resulted in an initial DODAG formation time of 22 seconds, with full data relay completion within 180 seconds of formation. This implementation demonstrates HOIST operating in an environment with uncontrolled mobility and frequent loss of communications between the Bridges and the Messenger.

Further validation testing was completed by simulation.

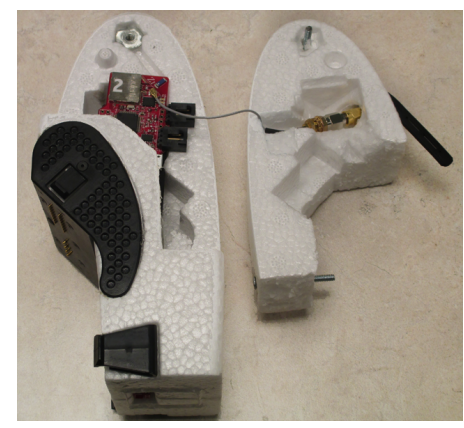

Figure 11: Payload Bay on Skate UAS

We modified the simulation in Cooja by increasing the Collector size to 32 , with 8 Bridges. Our mobility model brings the Messenger within range after 30 seconds of simulation time. The Messenger transits, back and forth, between the two end Bridges for the duration of the simulation, at a speed of $5.0 \mathrm{~m} / \mathrm{s}$. Over 60 simulations, we found that using a hop-count based objective function was more effective at this rate of mobility for an orbiting mobile sink. Future work will be done analyzing efficacy using UAS platforms.

\subsection{Data Analysis Conclusion}

After looking at the results of the data analysis, we have selected to use a minimum DIO send time of $2.048 \mathrm{~s}$, with 6 doublings, and a 2 second send rate for future implementation testing. These timings, while relating particularly to the RPL routing protocol, enable us to effectively assess HOIST under various conditions. These assessments of HOIST operating under various terrestrial and aerial mobility models provided validation that HOIST is both effective at retrieving data from remote field and is tenable when increasing the size of the number of Bridge and Collector devices.

\section{CONCLUDING REMARKS}

This is an initial proposal and a basic implementation that is not without discovered limitations on the proposed architecture. We seek to continue our work and aim to refine and present a fully operational HOIST for consideration as a viable mobility-aware, hierarchical solution that can run on off-the-shelf hardware, using a common routing protocol and a simple application-level implementation. These factors allow HOIST to handle high loss rates, instability, and low data rate transfers through both data retrieval from geographically segregated fields and local field, spot-checking operations.

While we are continuing to enhance our implementation of HOIST as described in this paper, our current focus is on using HOIST as a platform for exploring mobility in WSNs. One aspect of this is working with mobility-awareness within the RPL routing protocol. In our work, we have explored timings for RPL route maintenance messages and we are using RPL's Instance structure to provide HOIST with information concerning the presence of the mobile sinks; however, RPL has difficulty in modifying routing in response to mobility, once a route is formed. We are continuing to explore the underlying factors so that we may improve our response to mobility.

HOIST has the ability to adjust the number of data blocks 
to send before requesting confirmation; we are working with extending this component to also react to the particular model of mobility in use. An orbiting aerial data relay will have different optimal data transmission patterns than a small autonomous ground vehicle that is able to stop until all data transfers are complete. As we have seen in flight testing, even when the mobility profile is known, external factors, such as high wind conditions, can prevent the expected behavior. Mobility assessment and prediction can enable HOIST, or even RPL, to adjust by changing objective functions, proactively changing routing assignments, or even invalidating the entire network, prompting a global rebuilding.

This paper presents several motivating desires and provides the HOIST architecture to address those needs. HOIST addresses issues with disproportionate energy-drain and managing geographically segregated deployment areas by leveraging the innate benefits of a hierarchical network model. HOIST also uses basic mobility-aware systems, such as the modified lifetime, in order to responsively react to the presence of both Messenger and Observer sinks.

\section{ACKNOWLEDGEMENT}

This material is based upon work supported by the National Science Foundation under Grant No. CNS-1116122 and CNS-1205453.

\section{REFERENCES}

[1] T. Winter et al., RPL: IPv6 Routing Protocol for Low-Power and Lossy Networks, RFC 6550 (Proposed Standard), Internet Engineering Task Force Std. 6550, Mar. 2012.

[2] J. Valente et al., "An air-ground wireless sensor network for crop monitoring," Sensors, vol. 11, no. 6, pp. 6088-6108, 2011.

[3] N. Grammalidis et al., "A multi-sensor network for the protection of cultural heritage," in 19th European Signal Processing Conference, 2011, Aug 2011, pp. 889-893.

[4] A. Somova et al., "Deployment and evaluation of a wireless sensor network for methane leak detection," Sensors and Actuators A: Physical, vol. 202, pp. 217-225, Nov. 2013.

[5] T. Watteyne et al., "From manet to ietf roll standardization: A paradigm shift in wsn routing protocols," Communications Surveys Tutorials, IEEE, vol. 13, no. 4, pp. 688-707, Fourth 2011.

[6] E. Ancillotti, R. Bruno, and M. Conti, "Reliable data delivery with the ietf routing protocol for low-power and lossy networks," IEEE Transactions on Industrial Informatics, vol. 10, no. 3, pp. 1864-1877, Aug 2014.

[7] M. Di Francesco, S. K. Das, and G. Anastasi, "Data collection in wireless sensor networks with mobile elements: A survey," ACM Trans. Sen. Netw., vol. 8, no. 1, pp. 7:1-7:31, Aug. 2011.

[8] Y. Gu, F. Ren, Y. Ji, and J. Li, "The evolution of sink mobility management in wireless sensor networks: A survey," IEEE Communications Surveys Tutorials, vol. PP, no. 99, pp. 1-1, 2015.

[9] J. Lloret et al., "A cluster-based architecture to structure the topology of parallel wireless sensor networks," Sensors, vol. 9, no. 12, p. 10513, 2009.
[10] P. Ding, J. Holliday, and A. Celik, "Distributed energy-efficient hierarchical clustering for wireless sensor networks," in Proceedings of the First IEEE International Conference on Distributed Computing in Sensor Systems, ser. DCOSS'05. Berlin, Heidelberg: Springer-Verlag, 2005, pp. 322-339.

[11] C.-C. Huang et al., "Hierarchical role-based data dissemination in wireless sensor networks," The Journal of Supercomputing, vol. 66, no. 1, pp. 35-56, 2013.

[12] C.-J. Lin, P.-L. Chou, and C.-F. Chou, "Hcdd: Hierarchical cluster-based data dissemination in wireless sensor networks with mobile sink," in Proceedings of the 2006 International Conference on Wireless Communications and Mobile Computing, ser. IWCMC '06. New York, NY, USA: ACM, 2006, pp. 1189-1194.

[13] R. Shah et al., "Data MULEs: modeling a three-tier architecture for sparse sensor networks," in Proceedings of the First IEEE International Workshop on Sensor Network Protocols and Applications, 2003., May 2003, pp. 30-41.

[14] M. Zhao and Y. Yang, "A framework for mobile data gathering with load balanced clustering and mimo uploading," in INFOCOM, 2011 Proceedings IEEE, April 2011, pp. 2759-2767.

[15] Y. Yang et al., "Opportunities for wsn for facilitating fire emergency response," in 5th International Conference on Information and Automation for Sustainability (ICIAFs), 2010, Dec 2010.

[16] S. Basagni, A. Carosi, and C. Petrioli, "Controlled vs. uncontrolled mobility in wireless sensor networks: Some performance insights," in IEEE 66th Vehicular Technology Conference, 200\%. VTC-2007 Fall., Sept 2007, pp. 269-273.

[17] M. Perillo, Z. Cheng, and W. Heinzelman, "An analysis of strategies for mitigating the sensor network hot spot problem," in The Second Annual International Conference on Mobile and Ubiquitous Systems: Networking and Services, 2005. MobiQuitous 2005., July 2005, pp. 474-478.

[18] D. Carels et al., "Support of multiple sinks via a virtual root for the rpl routing protocol," EURASIP Journal on Wireless Communications and Networking, vol. 2014, no. 1, 2014.

[19] O. Gaddour et al., "Co-rpl: Rpl routing for mobile low power wireless sensor networks using corona mechanism," in 9th IEEE International Symposium on Industrial Embedded Systems (SIES), 2014, June 2014, pp. 200-209.

[20] V. Safdar et al., "A hybrid routing protocol for wireless sensor networks with mobile sinks," in 7th International Symposium on Wireless and Pervasive Computing (ISWPC), 2012, July 2012, pp. 1-5.

[21] G. E. P. Box and M. E. Muller, "A note on the generation of random normal deviates," Ann. Math. Statist., vol. 29, no. 2, pp. 610-611, 061958.

[22] P. Levis et al., The Trickle Algorithm, RFC 6206 (Standards Track), Internet Engineering Task Force Std. 6206, Mar. 2011.

[23] "Skate." [Online]. Available: http://www.aurora.aero/products/skate.aspx 